\title{
A Novel Approach for Privacy Policy on Online Social Network
}

\author{
Prof. P.S. Hanwate ${ }^{1}$, Pradnya Kapile ${ }^{2}$, Kajal Katariya ${ }^{3}$, Sunny Yadav $^{4}$, Ranjeet Patil ${ }^{5}$ \\ Professor, Computer Dept., NBNSSOE, Pune, India ${ }^{1}$ \\ Student, Computer Dept., NBNSSOE, Pune, India ${ }^{2,3,4,5}$
}

\begin{abstract}
Photograph sharing is an alluring element which advances on line Social Networks (OSNS). Tragically, it could release clients' privateness in the event that they're permitted to distribute, comment, and tag a photo uninhibitedly. we endeavor to address this issue and observe the situation while a man offers a photo containing people other than him/her (named co-picture for brief).To spare you suitable privateness spillage of a photo, we format a component to permit every person in a picture know about the posting interest and take an interest inside the decision making at the photograph posting. For this rationale, we require a productive facial acknowledgment (FR) machine which can catch every one of us inside the photo. In any case, additional distressing privateness putting may furthermore limitation the assortment of the pictures openly accessible to instruct the FR contraption. To address this dilemma, our component endeavors to use clients' private pics to plan a customized FR contraption specifically prepared to recognize feasible picture co-proprietors without releasing their privateness. We moreover widen designated agreement based method to diminish the computational multifaceted nature and monitor the individual tutoring set. We demonstrate that our gadget is better than other reasonable strategies in expressions of notoriety proportion and execution. Our component is executed as confirmation of idea Android programming on Face book's stage. The vitality direction dissemination is brought about by the particular join method, in which the likelihood of a man An associating with a client B is corresponding to the scope of B's present associations. Watchwords: Social system, photograph protection, secure multi-party calculation, bolster vector machine, collective learning.
\end{abstract}

Index Terms: Social network, photo privacy, secure multi-party computation, support vector machine, collaborative learning.

\section{INTRODUCTION}

As of late we can give a couple security secret key which might be hacked and might be utilized by the others and furthermore we will share any photo as we adore on OSNs, regardless of whether this photograph incorporates diverse individuals (is a co-picture) or at no time in the future. as of now there is no restriction with sharing of co-photographs, at the opposite, social group bearer organizations like Face book are urging clients to submit co-pics and tag their buddies that enables you to get more people stressed. Picture sharing is an alluring trademark which promotes on-line Social Networks (OSNs). Tragically, it might release clients' protection on the off chance that they're permitted to set up, remark, and tag a picture unreservedly.

On this paper, we endeavor to adapt to this issue and watch the circumstance while a customer stocks a photo containing individuals separated from himself/herself (named co-photo for short). To spare you conceivable protection spillage of a photo, we design an instrument to allow each man or lady in a photo know about the posting pastime and take an interest inside the choice making on the photograph posting. Thus, we require a productive facial notoriety (FR) machine that can see every individual inside the photo. In any case, additional irritating security setting may furthermore restrain the wide assortment of the previews freely accessible to teach the FR contraption. To manage this problem, our system endeavors to make utilization of clients' close to home pictures to format a redid FR gadget specifically gifted to separate attainable photograph co-proprietors without releasing their privateness.

We also create distributed agreement based procedure to reduce the computational multifaceted nature and shield the private tutoring set. We demonstrate that our machine is progressed to other doable strategies as far as notoriety proportion and execution. Our component is done as a proof of thought Android programming on Face digital book's stage. Social locales have end up being vital a piece of our regular presence. on-line informal organizations (OSNS) which incorporate face digital book, Google and sound of winged creatures are intrinsically intended to make capable individuals to part private and open measurements and make social associations with companions, co-individuals, individuals having like-part, possess family, or even with outsiders. To hold secure (out of risk) individual data, path in control has become a prime issue variable of OSNS. In any case, it will end up plainly everlasting record when some 
photo/picture is posted/transferred. Past due outcomes can be unsafe; individuals may utilize it for exceptional sudden capacities. A movement is needed to over these many issues of informal organizations and makes the interpersonal organizations exceptionally casual.

\section{RELATED WORK}

1. N. Mavridis, w. Kazmi, and p. Toulis. buddies with faces: how Social networks can beautify face popularity and vice versa. In Computational social community evaluation, computer communications and networks, pages 453-482. Springer London, 2010. study the records of photograph Sharing on social networks and suggest a three realms model: "a social realm, wherein identities are entities, and friendship a relation; $2 \mathrm{~d}$, a visible sensory realm, Of which faces are entities, and co-prevalence in pix A relation; and third, a physical realm, in which bodies Belong, with physical proximity being a relation." They show that any nation-states are pretty correlated. Given facts in a single realm, we are able to give an amazing Estimation of the connection of the other realm.

2. Z. Stone, t. Zickler, and t. Darrell. toward big-scale face recognition using social community context. court cases of the ieee, ninety eight(8):1408-1415., z. Stone, t. Zickler, and t. Darrell. Autotagging fb: Social network context improves photo annotation. In computer vision and sample recognition workshops, 2008. Cvprw'08. Ieee pc society conference on, pages 1-8. Ieee, 2008.advocate to use The contextual statistics inside the social realm and cophoto dating to do computerized fr. They define a Pairwise conditional random area (crf) version to locate The top-quality joint labeling via maximizing the conditional Density. especially, they use the present classified pics as the education samples and combine the photo cooccurrence information and baseline fr rating to enhance The accuracy of face annotation.

3. k. Choi, h. Byun, and ok.-a. Toh. A collaborative face popularity Framework on a social network platform. In automated face gesture reputation, 2008. Fg '08. eighth ieee global convention on, Pages 1-6, 2008.

speak the distinction between the traditional fr system and the Fr gadget this is designed specifically for osns. They point out that a custom designed fr gadget for each person is predicted to be an awful lot greater accurate in his/her own picture Collections.

4. J. Y. Choi, w. De neve, k. Plataniotis, and y.-m. Ro. Collaborative Face recognition for improved face annotation in personal photo Collections shared on online social networks. Multimedia, ieee Transactions on, 13(1):14-28, 2011.

Propose to use multiple personal fr engines to Work collaboratively to improve the recognition ratio. Specifically, they use the social context to select the suitable Fr engines that contain the identity of the queried Face image with high probability.

5. D. Rosenblum. What each person can recognize: the privacy risks of social Networking web sites. protection privacy, ieee, five(3):40-forty nine, 2007. The privateness leakage caused by The terrible get entry to control of shared facts in web 2.0 is properly studied.

6. C. Squicciarini, m. Shehab, and f. percent. Collective privacy management In social networks. In proceedings of the 18th international convention on global wide internet, www '09, pages 521-530, big apple, big apple, u.s.a., 2009. Acm. Propose a recreation-theoretic scheme wherein the privateness rules are collaboratively enforced over the shared statistics. Each person Is capable of outline his/her privacy coverage and publicity policy. Handiest while a picture is processed with owner's privateness coverage and co-owner's publicity coverage could it be published.

\section{IMPLEMENTATION}

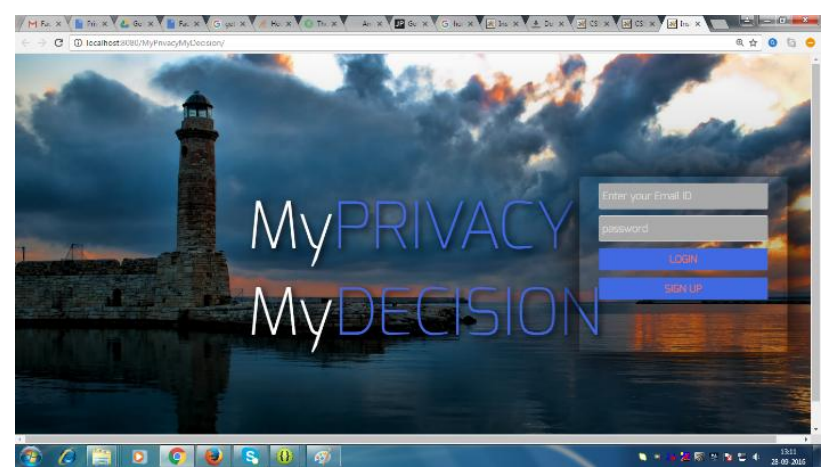

Fig. 1 


\section{IJARCCE}

User need to register to application by filling all register details. After registering, user will get login credentials i.e. username and password. User can login to application by entering user name and password. Home page will be displayed as shown in fig. 1. Registration form is shown in fig. 2

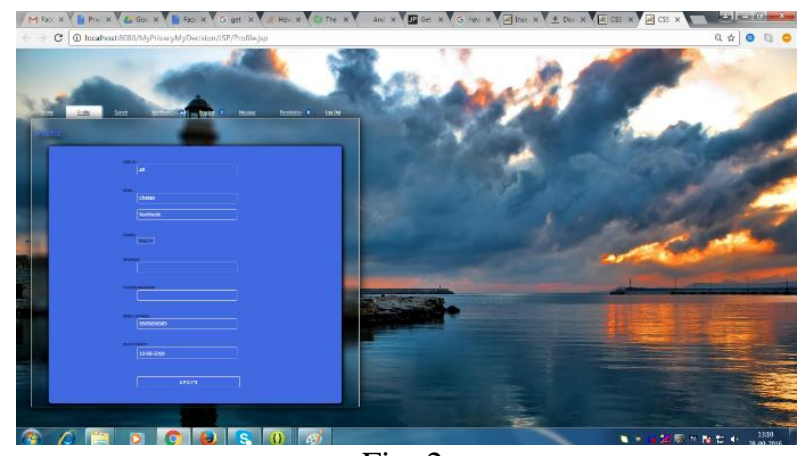

Fig. 2

After successful login, user will get OTP. User will enter OTP and further process will be carried out as shown in fig. 3

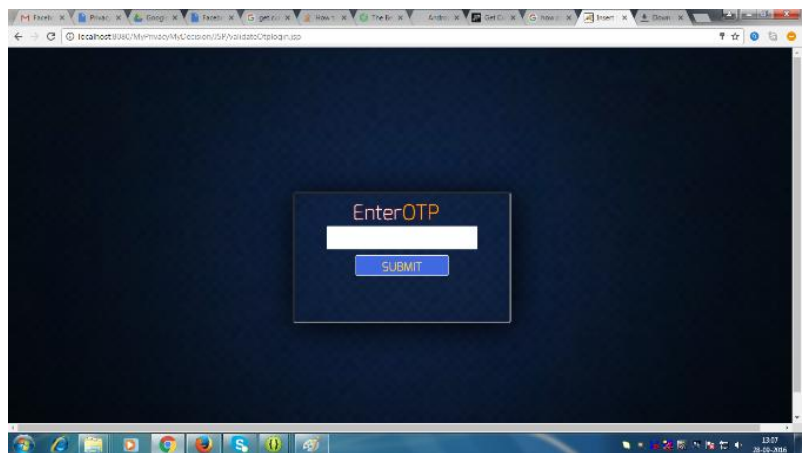

Fig. 3

Our main aim is face recognition system. Uploaded photo will store in database. . FR system provides privacy by notifying the subject about the posting activity and thus leading the other subjects to take active part in it. To prevent possible privacy leakage of a photo, we design a mechanism to enable each individual present in a photo be aware of the posting activity and participate in the decision making on the photo posting. For this purpose, an efficient facial recognition (FR) system is needed which recognizes everyone in the photo. However, if more privacy settings are done then it may bind the number of photos necessary to train the FR system. So in order to solve this problem, private photos of users is utilized to train the FR system and thus prevent the leakage of the privacy of the individuals.

It is shown in fig. 4

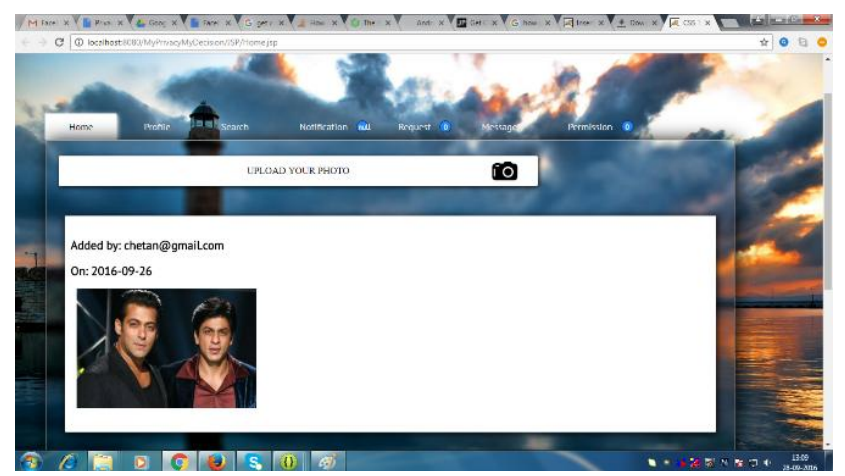

Fig. 4

\section{CONCLUSION}

Photograph sharing is a standout amongst the most well known components in online informal communities, for example, Facebook. Lamentably, imprudent photograph posting may uncover protection of people in a posted 


\section{IJARCCE}

photograph. To control the protection spillage, we proposed to empower people conceivably in a photograph to give the consents before posting a co-photograph. We composed a protection saving FR framework to recognize people in a cophotograph. The proposed framework is highlighted with low calculation cost and classification of the preparation set. Hypothetical investigation and analyses were directed to show adequacy and proficiency of the proposed plot.

\section{REFERENCES}

1. N. Mavridis, w. Kazmi, and p. Toulis. Friends with faces: how Social networks can enhance face recognition and vice versa. In Computational social network analysis, computer communications And networks, pages 453-482. Springer london, 2010.

2. Z. Stone, t. Zickler, and t. Darrell. Toward large-scale face Recognition using social network context. Proceedings of the ieee, 98(8):14081415., z. Stone, t. Zickler, and t. Darrell. Autotagging facebook: Social network context improves photo annotation. In computer Vision and pattern recognition workshops, 2008. Cvprw'08. Ieee Computer society conference on, pages 1-8. Ieee, 2008.

3. K. Choi, h. Byun, and k.-a. Toh. A collaborative face recognition Framework on a social network platform. In automatic face gesture Recognition, 2008. Fg '08. 8th ieee international conference on, Pages 1-6, 2008.

4. J. Y. Choi, w. De neve, k. Plataniotis, and y.-m. Ro. Collaborative Face recognition for improved face annotation in personal photo Collections shared on online social networks. Multimedia, ieee Transactions on, 13(1):14-28, 2011.

5. D. Rosenblum. What anyone can know: the privacy risks of social Networking sites. Security privacy, ieee, 5(3):40-49, 2007.

6. C. Squicciarini, m. Shehab, and f. Paci. Collective privacy management In social networks. In proceedings of the 18 th international Conference on world wide web, www '09, pages 521-530, new York, ny, usa, 2009. Acm.

7. K.-B. Duan and S. S. Keerthi. Which is the best multiclass svm method? an empirical study. In Proceedings of the 6th international conference on Multiple Classifier Systems, MCS'05, pages 278-285,Berlin, Heidelberg, 2005. Springer-Verlag. 